\title{
Current status and challenge in clinical work of autism spectrum disorders in China
}

\author{
Zhi-Wei Zhu' ${ }^{1} \cdot$ Yan Jin ${ }^{1} \cdot$ Ling-Ling Wu ${ }^{1} \cdot$ Xiao-Lin Liu ${ }^{1}$
}

Received: 24 April 2018 / Accepted: 21 May 2018 / Published online: 12 June 2018

(c) Children's Hospital, Zhejiang University School of Medicine 2018

Autism spectrum disorders (ASDs) are a group of neurodevelopmental disorders featured by social communication and interaction deficits, and with limited interests and repetitive behaviors. In China, ASDs was first reported by Tao from Nanjing Brain Hospital in the early 1980s [1, 2]. Since then, more children with autism have been diagnosed. While the past more than 30 years have witnessed steady growth in ASD research and clinical diagnosis and intervention in China, there are still many challenges to overcome. In this article, we will present the current status of prevalence, screening and diagnosis of ASD in China and propose further efforts for practitioners and policy-makers to secure optimal outcomes for children with ASD.

During the past three decades, many population-based epidemiological studies have been available in China, but most of them were regional studies with relatively small sample sizes and the reported ASD prevalence varied widely. Li et al. conducted a national study in 2011, but they reported a very low prevalence of ASD as 2.38/10,000 with a male to female ratio of 2.01 , based on the data from the Second China National Sample Survey on Disability [3]. Recently, several researches were carried out to analyze the estimated prevalence of ASD using meta-analysis. In 2013, Sun et al. assessed the prevalence of childhood autism in China including Hong Kong and Taiwan regions based on 18 epidemiological studies from 1987 to 2011 . They estimated the pooled prevalence of autism in children as $11.8 / 10,000$ in the Mainland of China and 26.6/10,000 in all of China [4]. In the same year, a meta-analysis of the studies (excluding the only nationwide study) reported a pooled estimated prevalence of ASD of 24.5/10,000 in the Mainland of China

Zhi-Wei Zhu

zhuzhiwei@zju.edu.cn

1 Department of Developmental and Behavioral Pediatrics, Children's Hospital, Zhejiang University School of Medicine, No. 3333 Binsheng Road, Hangzhou 310052, China
[5]. In 2017, Shi et al. reported that the pooled prevalence of ASD among children aged 0-6 years during 2006-2015 was $35.1 / 10,000$, with a male to female ratio of 2.59:1 [6]. All the reported prevalence of ASD in China was much lower than those of many other countries, such as USA, UK and Korea, where the prevalence of ASD was higher than $1 \%$. Scholars working actively in the field suspect that the prevalence is underestimated in China; the main reason may be lacking qualified practitioners who can diagnose ASD.

Most institutions in the world currently make the diagnosis of ASD by administering both a play-based assessment (Autism Diagnostic Observation Schedule, ADOS) and a parent interview (Autism Diagnostic Interview-Revised, ADI$R$ ). In the past decade, more and more pediatric psychiatrists and psychologists and pediatricians in developmental and behavioral departments (DBP) in China (including our team) use ADOS and/or ADI-R to diagnose ASD. But most clinicians make the diagnosis according to a clinical judgment with international diagnostic criteria without any diagnostic assessment [7]. Some clinicians consider the screening results to be the final diagnosis without using any additional diagnostic instruments [8]. Some adopted the Childhood Autism Rating Scale (CARS) as the diagnostic instrument [4].

The screening, diagnosis and intervention process of ASD usually includes the following steps in our department: (1) primary health care practitioners across Zhejiang Province will monitor growth and development of children during the well-child visits. They will suggest further assessment if the child is delayed in motor, language and/or social skills. Children suspected with developmental delay or ASD will be taken to our department for further assessment; (2) pediatricians in our department interview the parents to get the message of symptoms of concern and some basic information about the child's developmental history; (3) then the doctor may conduct a structured observation and apply some basic screening assessments on the child to evaluate whether the concerns are valid or not. The Ages \& Stages Questionnaires (ASQ) and ASQ: Social-Emotion (ASQ-SE) 
will be used to screen the following areas: communication skills, motor skills, cognitive abilities, adaptive behaviors and social-emotional skills. And the Modified Checklist for Autism in Toddlers Revised with Follow-Up (M-CHAT-R/F) or the Autism Behavior Checklist (ABC) is used to screen ASD; (4) based on the screening results, if a red flag shows there are valid concerns, we will make an official diagnosis according to the diagnostic criteria or make an appointment for ADOS and/or ADI-R assessment if possible; (5) the children diagnosed with ASD will be assessed with other scales such as Psychological Educational Profile 3rd Edition (PEP-3) and Griffiths Development Scales Chinese Edition or Wechsler's Intelligence Scale IV to collect the information about the abilities of multi-domains. After assessments, we will discuss with the parents about the individual educational plan for their children. We provide periodical training lectures to the parents and interventions to the children.

However, not all children with ASD in China can get diagnostic processes as mentioned above. Only psychiatrists, and clinical psychologists or DBP pediatricians in major cities (e.g., Beijing, Shanghai, Guangzhou, Hangzhou, etc.), can make such a diagnosis [9]. Parents often take their children with concerns to local hospitals to see a pediatrician first. Unfortunately, most doctors at the local hospitals are not specialized in ASD diagnosis and cannot identify the problem. Suspected children will then be referred to other mental health professionals (such as DBP pediatricians, clinical psychologists or psychiatrists) in bigger cities. In most cases, the duration for diagnosing ASD is very long. So an accurate diagnosis of ASD in China usually depends on the professionals' competence and credentials, as well as on the parents' income and persistency [9].

The number of hospitals which are qualified to diagnose ASD increases rapidly these years in China. The study in Beijing Stars and Rain Institute for Autism (SR) found that the number of hospitals where the SR children got their diagnoses of ASD or "autistic tendencies" went up steadily over time, from just several hospitals in the early 1990s to 267 hospitals in 2012 [10]. And especially, the number of hospitals in sub-provincial cities and prefecture-level cities increased rapidly. Between 2007 and 2012, about 50\% diagnoses were made by hospitals in municipalities, a quarter in sub-provincial cities and about a fifth in prefecturelevel cities [10]. 56.4\% of these hospitals were located in the North, East, and Northeast of China, and with the most rapid increase in the well-developed East of China. By contrast, the number of hospitals in the less-developed Western and Central areas grows slowly [10].

In the past decade, a wider variety of hospitals began to make diagnoses. In addition to psychiatric hospitals, more and more children's hospitals, women and children healthcare hospitals and general hospitals can make diagnoses. And now most parents prefer to take their young child to a children's hospital or women and children healthcare hospital rather than to a psychiatric hospital.

Screening and surveillance are crucial components to the early detection of ASD in children, which enables early interventions that provide the best chances for improved outcomes. Surveillance is a flexible, continuous, longitudinal process aimed at identifying concerns, and it should be performed at every well-child visit. In 2017, the "Expert consensus on early screening and early intervention for children with autism spectrum disorders" was published, which has promoted standard screening and intervention for children with ASD in China [11]. All infants, toddlers, preschoolers, and early elementary-aged children should be screened at regular intervals. And older school-age children with developmental concerns or who are struggling in school may also require testing by a psychologist or DBP pediatrician.

Despite recent advances, the research and clinical works for children with ASD remains relatively sparse in China. Key clinical issues include the need for (1) national-wide survey on prevalence using the same scales for screening and diagnosis; (2) screening scales for children with different ages, including infants, toddlers, preschoolers and school ages; (3) diagnostic scales development, evaluation, and implementation; (4) multidisciplinary diagnostic and treatment group (including child psychiatrist, pediatrician, neurologist, and other related professionals such as speech and language pathologist, or occupational therapist).

Except this editorial, the editor also includes several papers on ASD in the current issue. They include a personal view point which described a parent-centered approach to autism diagnosis in early childhood by Dr. Michael E. Mintz from USA [12]; a review article which reviewed therapeutic diets in autism spectrum disorder management by Dr. M. Gogou and G. Kolios from Greece [13]; and an original article investigating serum proteomic profiling for autism using magnetic bead-assisted matrix-assisted laser desorption ionization time-of-flight mass spectrometry by Dr. Chen YN and her colleagues from China [14].

Acknowledgements We want to thank our colleague Li-Fei Hu for expert advice and collaboration in writing this editorial. We thank all the colleagues in our department for dedication to the clinical practice.

Author contributions YJ, LLW and XLL looked up the literatures and wrote the initial draft. ZWZ reviewed and revised the manuscript. All the authors approved the final version.

Funding National Natural Science Foundation of China (no. 81373015).

\section{Compliance with ethical standards}

Conflict of interest All the authors disclosed no conflict of interest. 


\section{References}

1. Tao G. The problems of diagnosis and classification of infantile autism. J Chin Neuropsychiatry. 1982;2:104 (in Chinese).

2. Tao K. Infantile autism in China. J Autism Dev Disord. 1987;17:289-96.

3. Li N, Chen G, Song X, Du W, Zheng X. Prevalence of autismcaused disability amo Chinese children: a national populationbased survey. Epilepsy Behav. 2011;22:786-9.

4. Sun X, Allison C, Matthews FE, Sharp SJ, Auyeung B, BaronCohen S, et al. Prevalence of autism in mainland China, Hong Kong and Taiwan: a systematic review and meta-analysis. Mol Autism. 2013;4:7.

5. Wan Y, Hu Q, Li T, Jiang L, Du Y, Feng L, et al. Prevalence of autism spectrum disorders among children in China: a systematic review. Shanghai Arch Psychiatry. 2013;25:70-80.

6. Shi H, Zhang J, Zhang R, Wang X. Prevalence of autism spectrum disorders in children aged 0-6 years in China: a meta-analysis. Beijing Da Xue Xue Bao Yi Xue Ban. 2017;49:798-806 (in Chinese).

7. Li L, Li X, Wu W, Cai X, Fan X, Wei X, et al. Cross-sectional survey of autism spectrum disorders in children aged 0-6 years in Hainan province. Zhonghua Liu Xing Bing Xue Za Zhi. 2017;38:1187-90 (in Chinese).
8. Wong V, Hui S. Epidemiological study of autism spectrum disorder in china. J Child Neurol. 2008;23:67-72.

9. Huang AX, Jia M, Wheeler JJ. Children with autism in the People's Republic of China: diagnosis, legal issues, and educational services. J Autism Dev Disord. 2013;43:1991-2001.

10. Zhou W, Ye A, Sun Z, Tian H, Pu T, Wu Y, et al. Statistical analysis of twenty years (1993 to 2012) of data from mainland China's first intervention center for children with autism spectrum disorder. Mol Autism. 2014;5:52.

11. Developmental \& Behavioral Group of Chinese Academy of Pediatrics. Expert consensus on early screening and early intervention for children with autism spectrum disorders. Zhonghua Er Ke Za Zhi. 2017;55:890-7 (in Chinese).

12. Mintz ME. A parent-centered approach to autism diagnosis in early childhood. World J Pediatr. 2018. https://doi.org/10.1007/ s12519-018-0154-6.

13. Gogou M, Kolios G. Are therapeutic diets an emerging additional choice in autism spectrum disorder management? World J Pediatr. 2018. https://doi.org/10.1007/s12519-018-0164-4.

14. Chen YN, Du HY, Shi ZY, He L, He YY, Wang D. Serum proteomic profiling for autism using magnetic bead-assisted matrixassisted laser desorption ionization time-of-flight mass spectrometry: a pilot study. World J Pediatr. 2018. https://doi.org/10.1007/ s12519-017-0102-x. 